\title{
Interfacial Complexions and the Related Atomic Transport Behaviors in Gold- Oxide Systems
}

\author{
Guo-zhen Zhu ${ }^{1}$, Yong-sheng Fu ${ }^{1}$, Fang Liu ${ }^{1}$, An-chao Yi ${ }^{1}$, Dong Yue Xie ${ }^{1}$, and Wei Zhou ${ }^{1}$ \\ 1. State Key Laboratory of Metal Matrix Composites, School of Materials Science and Engineering, \\ Shanghai Jiao Tong University, Shanghai, 200240, P. R. China.
}

The interfacial complexions, referring to the quasi-two-dimensional "phase", have confined chemical and structural states associated with a set of thermodynamic parameters, e.g. temperature, pressure, and chemical potential. The formation and transition of interfacial complexions, particularly those at grain boundaries, have been closely related to a wide variety of atomic transport processes such as abnormal grain growth and activated sintering. [1] In spite of their importance for many fundamental scientific mysteries, the atomic/electronic structures of interfacial complexions as well as the related atomic transport properties remain largely unclear in literature, except a few grain boundary complexions reported in selected materials. Such problems can be more complicated due to tunable crystallographic features of their abutting bulk phases even for the same type complexion.

Recently, we discovered the formation of interfacial complexions between gold nanoparticles and oxide substrates, and an associated phenomenon of the spontaneous growth of nominally stable substrates underneath dewetted gold nanoparticles. Such phenomenon was repeatedly detected in $\mathrm{Au} / \mathrm{MgAl}_{2} \mathrm{O}_{4}$ [24], $\mathrm{Au} / \mathrm{TiO}_{2}$, and $\mathrm{Au} / \mathrm{SrTiO}_{3}$ systems. It should be noted that these materials have been investigated as potential heterogeneous catalysts for energy-related reactions. [5-6] Therefore, our study on the goldoxide interfacial complexions not only provides a new route of manipulating atomic transport behavior for nanostructure growth, but also extends our understanding of the fundamental theories about their catalytic behaviors.

This regrowth phenomenon occurs during the process of annealing gold overlayers on single-crystal oxide supports. The gold overlayers were sputtered on clean surfaces of selected oxide substrates. The annealing treatment was carried out over a temperature range of $600-1100^{\circ} \mathrm{C}$ for $10-90$ mins in highpurity Ar atmosphere. As shown in Fig.1, the dewetted Au nanoparticles, with a nearly Wulff equilibrium shape, have a few preferential crystallographic orientations with respect to the single-crystal substrate. Particularly within $\mathrm{Au} / \mathrm{MgAl}_{2} \mathrm{O}_{4}$ with a good lattice match, all detectable preferential orientations are proved to have twinned relationships. The regrowth oxide bases keep an ideal epitaxial relationship with the substrates. This regrowth phenomenon is related to a few parameters, such as the thickness of gold overlayer and annealing temperature.

More remarkably, we detected reconstructed interfacial monolayers, which have completely different atomic arrangement compared to both gold and oxide lattices. Taking $\mathrm{Au} / \mathrm{MgAl}_{2} \mathrm{O}_{4}$ as an example, the interfacial complexions include a gold-rich monolayer extending the spinel lattice. (see Fig.2) Depending on the annealing temperature, the interfacial complexions have different structures in addition to this gold-rich monolayer. The nucleation and migration of the interfacial complexions are related to the defects including the intersection points of twin planes at the interface. In the case of Au$\mathrm{TiO}_{2}$ with much larger lattice mismatch, more complicated interfacial structures have been recorded, and the detailed structures depend on annealing temperature and the crystallographic orientations between gold and rutile lattices. 
The structures of the interfacial complexions, e.g. detailed atomic arrangement and chemical bonding, are currently being investigated in order to shed light on understanding the related atomic transport behaviours.

\section{References:}

[1] S. J. Dullon et al, Acta Materialia 55(2007) p. 6208-6218.

[2] G.-z. Zhu, et al, Applied Physics Letters 105(2014), p. 231607.

[3] T. Majdi, et al, Applied Physics Letters 107(2016), p. 241601.

[4] F. Liu, et al, Materials Characterization 113(2016), p. 67-70.

[5] M. Haruta, et al, Jounral of Catalyst 144(1993), p. 175.

[6] C. H. Christensen, et al, Angewandte Chemie Internation Edition 45(2006), p. 4648.

[7] The authors acknowledge funding from the National Science Foundation of China (No. 51401124).

Ms. Tahereh Majdi is thanked for her many useful discussions and contributions to this work.
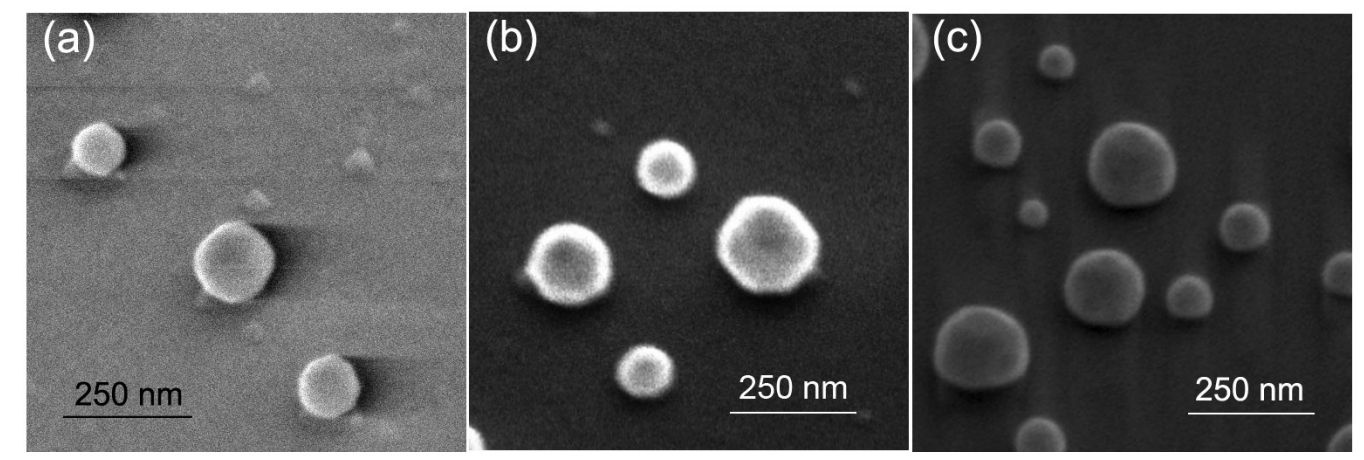

Figure 1. Intricately shaped gold-oxide nanostructures including the dewetted gold nanoparticles and regrowth oxide bases. (a), (b), and (c) are the SEM images from $\mathrm{Au}-\mathrm{MgAl}_{2} \mathrm{O}_{4}, \mathrm{Au}-\mathrm{SrTiO}_{3}$, and $\mathrm{Au}-$ $\mathrm{TiO}_{2}$, respectively.
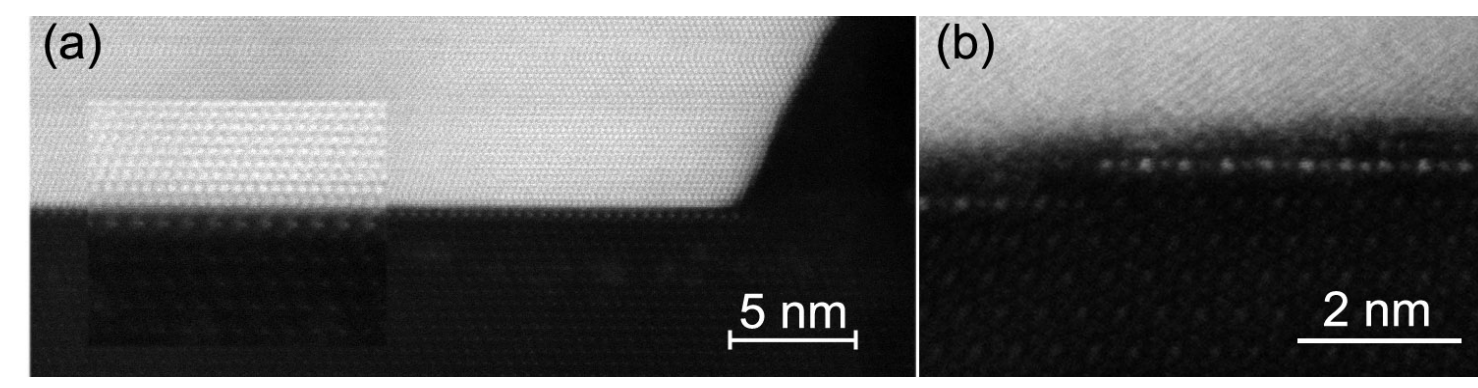

Figure 2. The interfacial complexions in the $\mathrm{Au}-\mathrm{MgAl}_{2} \mathrm{O}_{4}$ model system at different temperatures. The annealing temperature for (a) is higher than the one for (b). 